\title{
Cognitive Instruction Interface for Mobile Robot Navigation
}

\author{
Mohammed Elmogy, Christopher Habel, and Jianwei Zhang
}

\begin{abstract}
An essential aspect distinguishing robotics from other areas of artificial intelligent is their interaction with humans and their surrounding environments. Spatial knowledge can be represented in various ways to increase the interaction between human users and mobile robots. One effective way is to describe the route verbally to the robot. This method can permit inexpert users to instruct their mobile robots, which understand spatial descriptions, to naturally perform complex tasks using succinct commands.

We present a spatial language to describe route-based navigation tasks for a mobile robot. The instructions of this spatial language are implemented to provide an intuitive interface with which computer language naïve user can easily and naturally describe a navigation task to a mobile robot in any indoor environment. In our system, the instructions of the processed route are analyzed to generate a symbolic representation of the navigation task via the instruction interpreter. The resulting symbolic representation is used to generate a graphical representation of the route to supply the robot with the information about the route's environment and the relationships between the landmarks. It is also supplied to the robot motion planning stage as initial path estimation of route description and to ground the landmark symbols with their equivalent physical objects by using perceptual anchoring.
\end{abstract}

Index Terms - Natural language interfaces, navigation, and spatial reasoning.

\section{INTRODUCTION}

A more natural interaction between human users and mobile robots can be achieved by bridging the gap between the format of spatial knowledge used by robots and the format of spatial languages used by humans. This enables both sides to communicate by using common ground of understanding. Most typical scenarios of interaction between humans and robots include the user who instructs a robot to perform certain actions in certain scenarios, such as moving to a location or manipulating an object. To instruct the robot to

Mohammed Elmogy is with Department of Information Systems, Faculty of Computers \& Information Sciences, University of Mansoura, Egypt (email: melmogy@mans.edu.eg).

Christopher Habel is with Knowledge and Language Processing (WSV), Informatics department, University of Hamburg, Vogt-Kölln-straße 30, D22527 Hamburg, Germany. (email: habel@informatik.uni-hamburg.de).

Jianwei Zhang is with Technical Aspects of Multimodal Systems (TAMS), Informatics department, University of Hamburg, Vogt-Kölln-straße 30, D22527 Hamburg, Germany. (email: zhang@informatik.uni-hamburg.de). navigate in its surrounding environment, the robot navigation system should contain three basic components: planning process, navigation process, and environmental representation [3]. The planning process includes wayfinding and locomotion levels of navigation [4]. It computes a mobile robot path or trajectory between the start and end points of the route. The navigation process provides the robot with the information required to move and follow the computed path or trajectory and also to plan the footstep locations of the bipedal humanoid robots. Finally, the environmental representation enables the robot to know its location and direction during the navigation. In this paper, we will focus on the planning process and how to describe and analyze navigation tasks in a miniature city environment for a humanoid robot.

A more natural interaction between humans and mobile robots - with the least collective effort - can be achieved if there is a common ground of understanding $[1,2]$. A natural language interface supports more natural styles of interaction between robots and their users. Route descriptions are considered as one of the more important natural language interfaces between humans and mobile robots for applying an effective human-robot interaction.

To describe a navigation task to a mobile robot, route instructions are used to specify the spatial information about the route environment and the temporal information about the move and turn actions which will be executed by the robot [5]. Good route instructions should contain adequate information on these two aspects by considering the spatial environment of the robot and the relevant navigation and perception actions. To express the route in an effective way, the rules and sequence of commands should be expressed in very concisely. Natural language uses symbols and syntactic rules to interact with the robots which dispose of represented knowledge at the symbolic level.

On the other hand, spatial reasoning on the natural language route is essential for both humans and mobile robots. Spatial reasoning gives robots the ability to use human-like spatial language and provides the human user with an intuitive interface that is consistent with his innate spatial cognition [6]. It can also accelerate learning by using symbolic communication which has been shown in [7].

This paper is organized as follows. Section 2 discusses some current implementations of natural language interfaces for both mobile robots and simulated artificial agents. In section 3 , the structure of our route instruction language 
(RIL), which is used to describe the route for the mobile robot, is presented. The creation of the symbolic representation of the route is introduced in section 4 . Section 5 shows the topological map generation of the route's environment. The Grounding of the symbolic representation with the perceptual data in the physical environment is illustrated in section 6. Finally, the conclusion is presented in section 7 .

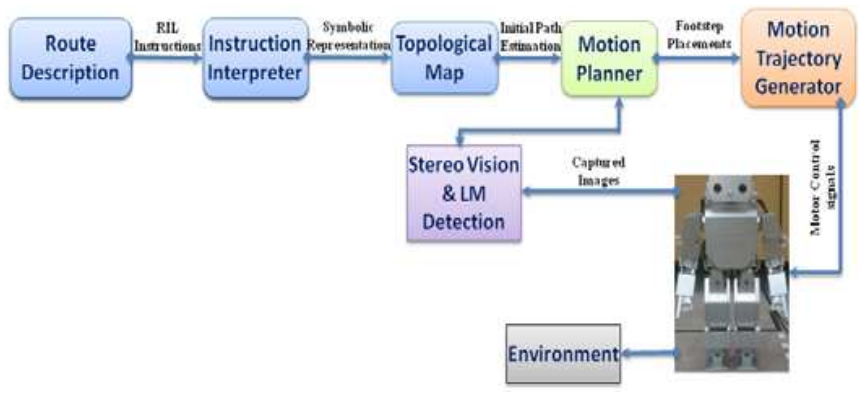

Fig. 1. System Architecture.

\section{RELATED WORK}

In the last three decades, there has been considerable research on spatial language and spatial reasoning. This motivates the research interest of using spatial language for interacting with artificial navigational agents. Many researchers $[6,8,9,10]$ have proposed frameworks using natural language commands in simulated or real-world environments to guide their artificial agents during navigation. In this section, some implementations of natural language interfaces for mobile robots and simulated agents will be discussed.

For simulated agents, Tschander et al. [8] proposed the idea of a Geometric Agent (GA) which simulates instructed navigation in a virtual planar environment. This geometric agent can navigate on routes in its virtual planer environment according to natural-language instructions presented in advance. In their approach, Conceptual Route Instruction Language (CRIL) is used to represent the meaning of natural language route instructions. It combines the latter with the spatial information gained from perception to execute the desired route. Tellex and Roy [9] implemented spatial routines to control the robot in a simulator. They defined a lexicon of words in terms of spatial routines and used that lexicon to build a speech controlled robot in a simulator. Their system is unified by a high level module that receives the output from the speech recognition system and simulated sensor data, creates a script using the lexicon and the parse structure of the command, and then sends appropriate commands to the simulated robot to execute that command. However, their current implementation acts only on the current snapshot of sensor readings which leads to errors in the robot's behavior.

On the other hand, there are considerable research efforts in developing various command sets for mobile robots and robotic wheelchairs [11-14]. The mobile robot community has created systems that can understand natural language commands. Many research efforts $[8,10,15,16]$ focus on using spatial language to control the robot's position and behavior, or to enable it to answer questions about what it senses. In general, previous work in this area has focused on developing various command sets for mobile robots and robotic wheelchairs, without directly addressing aspects of language that are context sensitive. Torrance [16] implemented a system that is capable of mediating between an unmodified reactive mobile robot architecture and domainrestricted natural language. He introduced reactive-odometric plans (ROPs) and demonstrates their use in plan recognition. The communication component of this architecture supports a typewritten natural language discourse with people. It lets users name places both directly or in relation to other known places, ask questions about the robot's plans and the spatial relationships of known places, and gives the robot short and long-term goals. This system was brittle due to place recognition from odometric data and the use of IR sensors for reactive motion control. Knowledge acquisition was concurrent with navigation, not prior to it. The resulting ROPs do not contain error-reducing stopping conditions, and this has caused problems in some parts of the tested environment where hallways do not sufficiently constrain the reactive navigation system.

Skubic et al. [10] implemented robot spatial relationships combined with a multimodal robot interface that provides the context for the human-robot dialog. They showed how linguistic spatial descriptions and other spatial information can be extracted from an evidence grid map and how this information can be used in a natural human-robot dialog. With this spatial information and linguistic descriptions, they established a dialog of spatial language. To overcome the object recognition problem (the system does not support vision-based object recognition), they have defined a class of persistent objects that are recognized and named by a human user.

In our system, we present a spatial language - called Route Instruction Language (RIL) [17] - to describe route-based navigation tasks for a humanoid robot. This language is implemented to present an intuitive interface that will be enable novice users to easily and naturally describe a route to a mobile robot in indoor and miniature city environments. The route description is parsed and processed via the instruction interpreter to generate a symbolic representation of the route description. The resulting symbolic script is used as an initial estimation of the robot path by the motion planner and also to generate a graphical representation of the route. Fig. 1 shows an overview structure of our system.

\section{ROUTE INSTRUCTION LANGUAGE (RIL)}

The RIL is developed to describe the route between the start and end points to a humanoid robot. It is intended as a semiformal language for instructing robots, to be used by nonexpert users via a structured graphical user interface. RIL provides elementary instruction statements which are processed to supply the robot with a sequence of motion 
actions. During navigation, this sequence of actions is processed by the motion planner to determine the footstep placements which will be effected by the humanoid robot to execute the route. Each statement in the RIL constitutes a spatial instruction which relates verbally coded motion concepts to one or more landmarks by use of a suitable spatial relationship. We used four types of basic actions in route instructions: moving from one place to another, turning or rotating in place, verifying a view description, and determining the current position. In route description, we use an extrinsic reference frame that is based on the robot's viewing perspective to describe all objects and directions with respect to the robot's body. The extrinsic reference is used to avoid ambiguity during route description and as a conversion process between different reference types.

TABLE I

RIL COMMAND SET AND SYNTAX

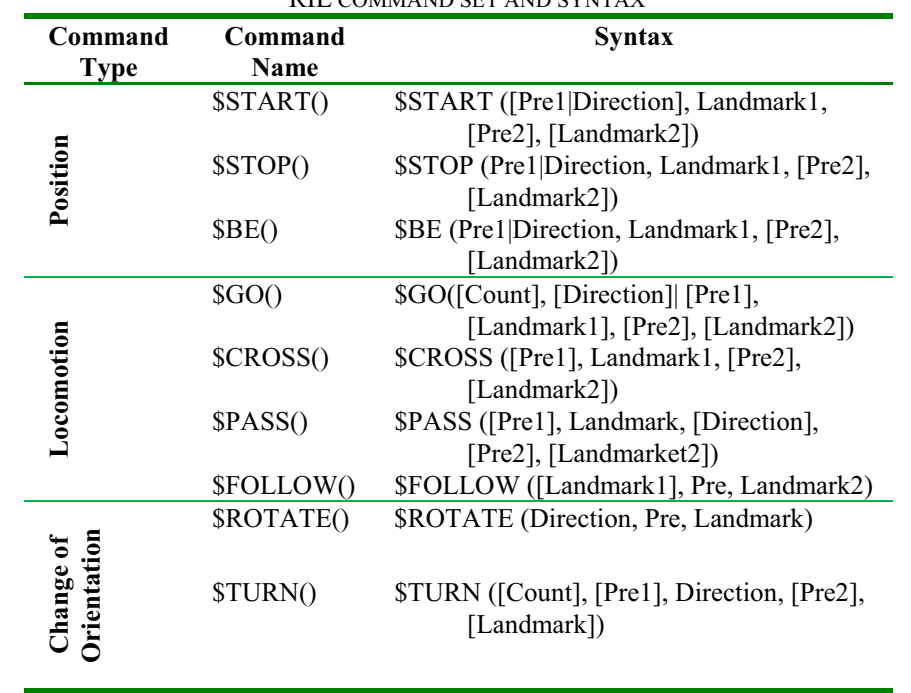

The commands of the RIL and their syntax are shown in Table I. Each instruction of the RIL specifies motion verbs, directions, destinations, and landmarks. The RIL commands are divided into three basic types: position, locomotion, and change of orientation commands. The position commands are used to indicate the current position of the robot during navigation. They are also used to determine the start and end points of the route. They consist of three commands: the \$START () command which is used to specify the beginning of the route with respect to specific landmark(s), the \$STOP() command which determines the position of the target point, and the $\$ B E()$ command which verifies the current robot position on the basis of one or more landmarks during navigation. Otherwise, locomotion commands are used to instruct the robot to move in the spatial environment in a specific direction or to follow a certain path. There are four different commands in this category. First, the $\$ \mathrm{GO}()$ command which orders the robot to move with respect to one or more landmarks, to move along a specific path, or to move with turning in a certain direction. Second, the \$CROSS() command which is used to let the robot cross a street or a corridor. Third, the \$PASS () command which is used to pass a landmark during motion to verify the position of the robot during navigation. Finally, the \$FOLLOW() command which is used to follow the boundaries of a landmark to reach a specific position. The last category is the change of orientation commands which consists of two commands: \$ROTATE() and \$TURN(). They are used to rotate around a landmark or turn in a certain direction, respectively.

The command syntax consists of a command word and an arbitrary number of arguments as shown Table I. The command word indicates the action which will be taken by the humanoid robot and is represented in the imperative form of the verb, e.g., GO, TURN, BE, etc. Each argument is a place holder for a specific group of words such as prepositions, directions, the number of turns, and landmarks. To add more flexibility to the command syntax, multiple kinds of command syntax have been defined. Mandatory arguments are typed without any brackets, whereas optional arguments are placed between rectangular brackets '[]'. The pipe symbol '|' indicates an OR operator. Fig. 2 shows an example of a route description from the town hall to the railway station in our miniature city using RIL.

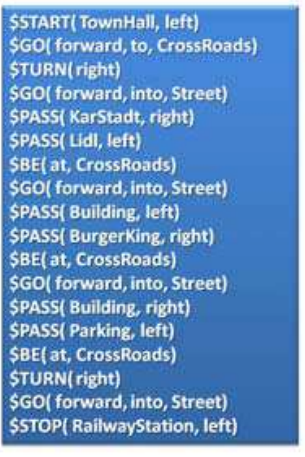

(a) Route Description

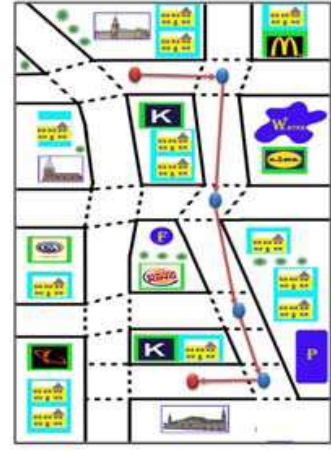

(b) Miniature City Map
Fig. 2. A route description from the town hall to the railway station in our miniature city using RIL.

We carried out an experiment to test the suitability of RIL for communicating a route description to a robot. 10 participants took part in the experiment (age 22 to 35 years). None of the participants had any background knowledge on route instructions and robotics. First, we gave them a description of the RIL syntax, a map of the miniature city, and an example of a suitable route description. We asked them to describe a route between the railway station and the McDonald's restaurant in the miniature city as depicted in Fig. 2(a). Most of them described the route correctly and $80 \%$ of the participants stated that the RIL is simple and easy to learn. $70 \%$ of the participants agreed that it is better to provide the commands of RIL with many optional parameters than to restrict them to a single syntax.

\section{INSTRUCTION INTERPRETER}

The instruction interpreter is used to discriminate, identify, and categorize the motion actions of the processed route description. It combines definitions from the lexicon according to the parse structure of the instruction, creating a 
symbolic script that describes the navigation process. The generated symbolic representation is used to create a topological map for the route environment. It is also supplied to the motion planner as an initial path estimation of the navigation task to help in generating the footstep placements for the humanoid robot. This symbolic script is based on the conceptual route instruction language (CRIL) which is developed by our group [8].

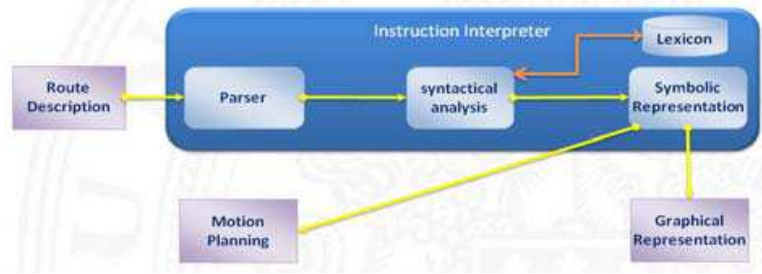

Fig. 3. The Instruction Interpreter Architecture.

The instruction interpreter contains a simple parser, a lexical analysis, and a syntactic analysis. The parser is supplied by the route description text. It separates the text into individual instructions. Each instruction is split into sequence of words using space and punctuation characters as delimiters. The resulting list is entered at the syntactical analysis stage to identify the structure of instructions by comparing their structure with a list of all kinds of instruction syntax which are understandable by the robot. Each word is looked up in the lexicon to obtain its type and features. The available types of words in the lexicon are command verbs, directions, prepositions, numbers of turns, and landmarks. Each verb entry in the lexicon consists of an action verb and an associated script composed from the set of its primitives and depends on the specified arguments passed to its instruction. It is defined as a script of primitive operations that run on data extracted from the analyzed instruction.

After analyzing the route instructions syntactically and connecting each resulting verb with its motion procedure, the symbolic representation of the route is generated. The resulting symbolic script consists of three basic components: motion actions, spatial relationships, and landmarks. The motion actions are classified into the following four different actions:

- BE_AT Action: It presents the position of the robot during navigation. It identifies the start, current, and end positions of the robot during navigation.

- GO Action: It indicates the motion actions which should be taken by the mobile robot.

- VIEW Action: It is used to notice a landmark in a certain direction or region during navigation.

- CH_ORIENT Action: It is used to indicate a change in the current orientation of the mobile robot motion during navigation based on a specific direction or landmark.

The spatial relationships are classified into two types. First, relations represent a location with respect to a landmark. Second, relations specify a direction with respect to one or two landmarks. Finally, the landmark features are retrieved from the knowledge base. They contain data about their shape, color or color histogram, and recognition method values. In addition to the retrieved features, the relationship feature is extracted from the processed route to describe the relation between the current processed landmark and other landmarks in the same path segment. Landmarks in our miniature city are classified into definite and indefinite landmarks depending on their features. Definite landmarks have unique characteristics which single them out from among the other landmarks in the miniature city, such as the Burger king restaurant, the Saturn store, and the town hall. On the other hand, indefinite landmarks have a number of properties that are not unique such as buildings, crossroads, and streets. Fig. 4 shows the resulted symbolic representation of the "town hall - railway station" route.

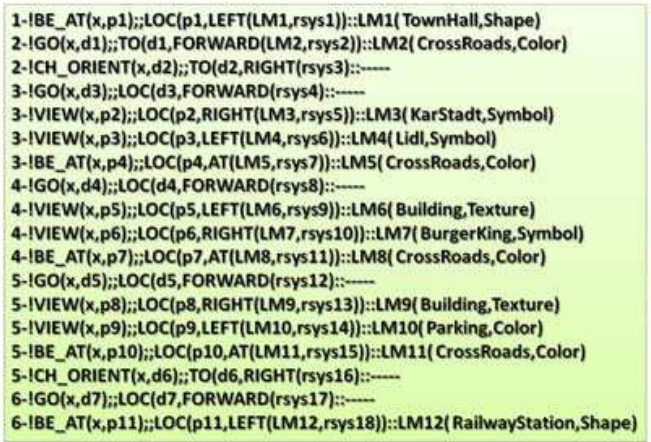

Fig. 4. The resulted symbolic representation of the "town hall - railway station" route description.

\section{GRAPHICAL REPRESENTATION}

When robots navigate in indoor environments, they require an adequate representation of the workspace. This representation should be abstract enough to facilitate higherlevel reasoning tasks like strategic planning or situation assessment, and still be detailed enough to allow the robot to perform lower-level tasks like path planning/navigation or self-localization [18]. Building a representation of the navigation workspace is an essential task for a mobile robot that aims to move autonomously in its surrounding space. The representation of spatial knowledge can be considered at two different levels of abstraction. On the one hand, metric (geometric) maps represent the environment according to the absolute geometric position of landmarks. On the other hand, a topological map describes relationships among features of the environment in a more abstract representation without any absolute reference system [19].

The topological map of the route, which represents a qualitative description of the robot's workspace, is generated from the resulting symbolic representation. It presents a graph-like description of the route where nodes correspond to significant, easy-to-distinguish landmarks, and arrows correspond to actions or action sequences which will be executed by the mobile robot. It represents only the interesting places, not the entire environment of the route. Fig. 5 shows the generated topological map of the route represented in Fig. 2. 


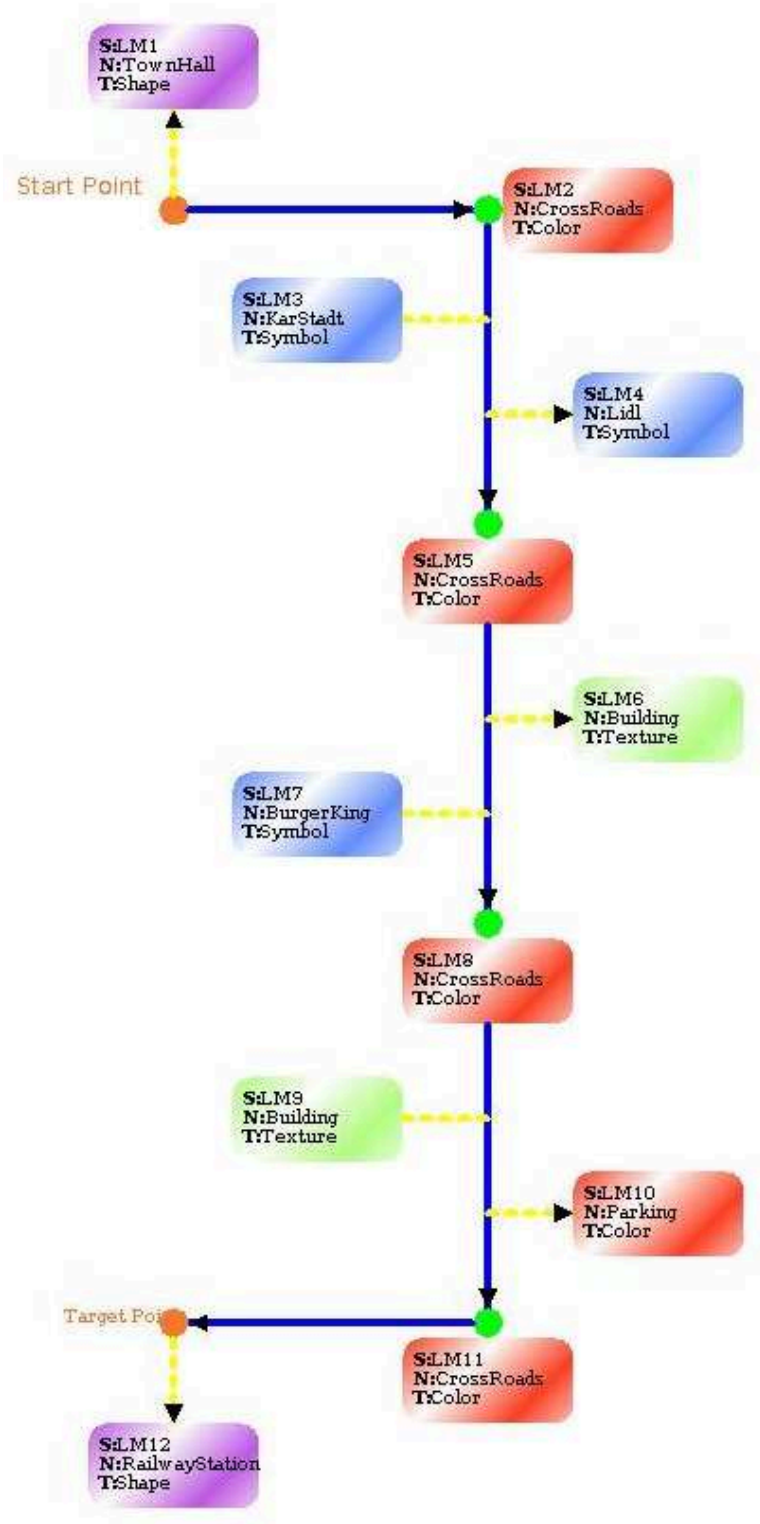

Fig. 5. Topological map of the "town hall - railway station" route.

In the topological map representation, the blue arrows represent the estimated robot path, whereas the yellow dashed lines represent the positions of the landmarks. The rounded rectangles represent the processed landmarks and their colors indicate the type of landmarks. Finally, orange circles indicate the start and destination points, whereas green circles represent intermediate nodes in the robot's path [20].

\section{SymbOl GROUNDING AND PERCEPTUAL ANCHORING}

After building the topological map, the resulting symbolic representation is supplied to the motion planner as an initial path estimation. The motion planner uses both the symbolic representation and the output of the stereo vision and landmark recognition stage to calculate the desired footstep placements of the humanoid robot to execute the processed route. The motion planner grounds the landmark symbols to their corresponding physical objects in the environment. Therefore, the symbolic and physical presentations of the landmarks should be integrated. Many researchers [21-23] have worked on the symbol grounding problem to solve the problem of incorporating the high-level cognitive processes with sensory-motoric processes in robotics. Cognitive processes perform abstract reasoning and generate planes for actions. They typically use symbols to denote objects. On the other hand, sensory-motoric processes typically operate from sensor data that originate from observing these objects. The researchers tried to maintain a coherence between representations that reflect actions and events, and the produced stream of sensory information from the environment. Accordingly, mobile robots need learning abilities that constrain abstract reasoning in relation to dynamically changing external events and the results of their own actions [6].

Harnard [21] considered perceptual anchoring as an important special case of symbol grounding. The anchoring is defined as the process of creating and maintaining the correspondence between symbols and sensor data that refer to the same physical objects [24]. We used a perceptual anchor to incorporate the symbols of the landmarks represented in the symbol system $(\Sigma)$ and the physical landmarks retrieved from the perceptual system ( $\Pi)$. The predicate grounding relation ( $\mathrm{g}$ ) is used to encode the correspondence between predicate symbols and admissible values of observable attributes. It contains the values of the landmark properties, such as color histogram values, shape, area range, and recognition method values. We used a color histogram, scale invariant features transform (SIFT), and the bag of features methods to recognize the landmarks [25]. As shown in Fig. 6, the perceptual anchoring $(\alpha)$ for a landmark contains a pointer to its symbol $(\sigma)$, a pointer to its physical object $(\pi)$, and its signature $(\gamma)$.

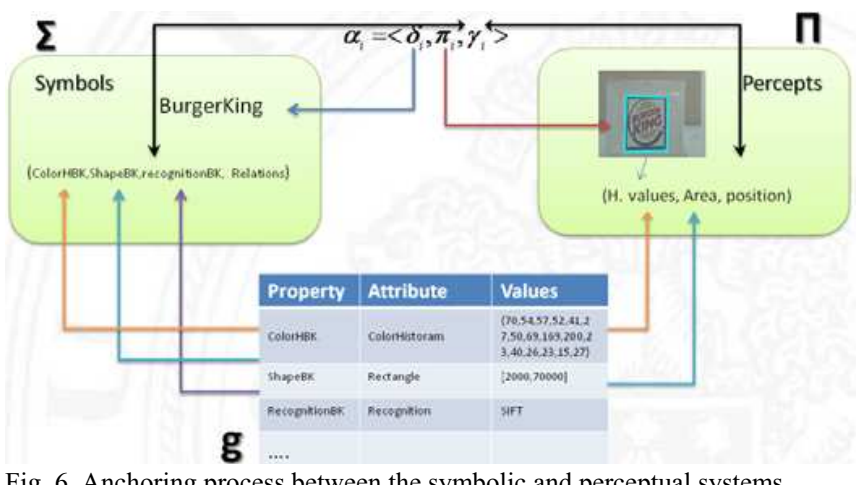

Fig. 6. Anchoring process between the symbolic and perceptual systems.

\section{CONCLUSION}

We presented The Route Instruction Language (RIL) - a semi-formal language - to be used by inexpert users to instruct humanoid robots in a miniature city environment. Based on RIL, we designed and realized an intuitive interface to mobile robots preventing misunderstanding and ambiguities 
in route descriptions. Starting from a set of commands, the instruction interpreter stage performs the analysis of route instructions and its lexicon relates the internal procedures to perceptual objects and specifies actions that can be carried out by the humanoid robot. The instruction interpreter analyzes the route to generate its equivalent symbolic representation which is supplied to the motion planner as initial path estimation.

The resulting symbolic representation of the route is used to generate a graphical representation of the route to supply the robot with global route information and prevent it from getting trapped in local loops or dead-ends in unknown environments. Finally, the symbolic representation is supplied to the motion planner to ground the landmark symbols to their equivalent physical objects by using perceptual anchoring.

\section{REFERENCES}

[1] S. Kiesler, "Fostering common ground in human-robot interaction," in proceedings of the IEEE international workshop on robot and human interactive communication (ROMAN), 2005, pp. 729-734.

[2] S. E. Brennan. "The grounding problem in conversations with and through computers," in Social and cognitive psychological approaches to interpersonal communication. Fussell SR \& Kreuz R J (Eds.). 1991. pp 201-225.

[3] G. Pradel, and P. Hoppenot, "Symbolic trajectory description in mobile robotics," Journal of Intelligent and Robotic Systems, vol.45, no. 2, February, 2006, pp. 157-180.

[4] D. R. Montello. Navigation. in The Cambridge handbook of visuospatial thinking. 2005. pp. 257-294.

[5] C. Habel, "Incremental generation of multimodal route instructions," in natural language generation in spoken and written dialogue, AAAI spring symposium 2003. palo alto, ca., 2003, pp. 44-51.

[6] S. Lauria, G. Bugmann, T. Kyriacou, J. Bos, and E. Klein, "Training personal robots using natural language instruction," IEEE Intelligent Systems, vol.16, 2001, pp. 38-45.

[7] A. Cangelosi, and S. Harnad, "The adaptive advantage of symbolic theft over sensorimotor toil: grounding language in perceptual categories,' Evolution of Communication, vol.4, 2000, pp. 117-142.

[8] L. B. Tschander, H. Schmidtke, C. Habel, C. Eschenbach, and L. Kulik. A geometric agent following route instructions. In Spatial cognition III, 2003. pp. 89-111.

[9] S. Tellex, and D. Roy, "Spatial routines for a simulated speechcontrolled vehicle," in proceedings of the 1st ACM sigchi/sigart conference on human-robot interaction, Salt Lake City, Utah, USA, 2006, pp. 156-163.

[10] M. Skubic, D. Perzanowski, S. Blisard, A. Schultz, W. Adams, M. Bugajska, and D. Brock, "Spatial language for human-robot dialogs,"
Systems, Man, and Cybernetics, Part C: Applications and Reviews, IEEE Transactions, vol.34, no. 2, May, 2004, pp. 154-167.

[11] R. Schulz, P. Stockwell, M. Wakabayashi, and J. Wiles, "Towards a spatial language for mobile robots," in proceedings of the 6th international conference on the evolution of language, , 2006, pp. 291298.

[12] S. Tellex, and D. Roy, "Grounding language in spatial routines," in proc. of AAAI spring symp. on control mechanisms for spatial knowledge processing in cognitive / intelligent systems, 2007.

[13] G. Pires, and U. Nunes, "A wheelchair steered through voice commands and assisted by a reactive fuzzy-logic controller," Journal of Intelligent and Robotic Systems, vol.34, 2002, pp. 301-314.

[14] R. C. Simpson, and S. P. Levine, "Adaptive shared control of a smart wheelchair operated by voice control," in proceedings of the 1997 IEEE/RSJ international conference on intelligent robots and systems (IROS '97), 1997, pp. 622-626.

[15] R. Bischoff, and T. Jain, "Natural communication and interaction with humanoid robots," in second international symposium on humanoid robots. tokyo, 1999 .

[16] M. C. Torrance. Natural communication with mobile robots. MIT Department of Electrical Engineering and Computer Science. 1994.

[17] M. Elmogy, C. Habel, and J. Zhang, "Spatial Language for Route-Based Humanoid Robot Navigation," in proceedings of the $4^{\text {th }}$ international conference on spatial cognition (ICSC2009), Roma, Italy, September, 2009.

[18] M. MacMahon, " Marco: a modular architecture for following route instructions," in Proceedings of the AAAI workshop on modular construction of human-like intelligence, Pittsburgh, PA, 2005, pp. 48-55.

[19] P. G. Zavlangas, and S. G. Tzafestas. Integration of topological and metric maps for indoor mobile robot path planning and navigation. Methods and applications of artificial intelligence. . 2002. pp. 121-130.

[20] M. Elmogy, C. Habel, and J. Zhang, "Robot topological map generation from formal route instructions," in proceedings of the 6th international cognitive robotics workshop at 18th european conference on artificial intelligence (ECAI), Patras, Greece, , 2008, pp. 60-67.

[21] S. Harnad, "The symbol grounding problem," Physica D. Nonlinear phenomena, vol.42, no. 1-3, June, 1990, pp. 335 - 346.

[22] A. Chella, S. Coradeschi, M. Frixione, and A. Saffiotti, "Perceptual anchoring via conceptual spaces," in Proc. of the AAAI-04 workshop on anchoring symbols to sensor data, 2004.

[23] L. Karlsson, A. Bouguerra, M. Broxvall, S. Coradeschi, and A. Saffiotti, "To secure an anchor - a recovery planning approach to ambiguity in perceptual anchoring," AI Communications, vol.21, no. 1, 2008, pp. $1-14$

[24] S. Coradeschi, and A. Saffiotti, " An introduction to the anchoring problem," Robotics and Autonomous Systems, vol.43, no. , , 2003, pp. 85-96.

[25] M. Elmogy, and J. Zhang, "Robust real-time landmark recognition for humanoid robot navigation," in proceedings of the 2008 IEEE international conference on robotics and biomimetics (ROBIO), Bangkok, Thailand, 2008 